Neurosurg Focus 13 (5):Article 1, 2002, Click here to return to Table of Contents

\title{
The role of cell therapy for stroke
}

\author{
Douglas Kondziolka, M.D., M.Sc., F.R.C.S.(C), LaWrence WechSLeR, M.D., \\ Elizabeth Tyler-Kabara, M.D., PhD., and Cristian AChim, M.D., Ph.D.
}

Departments of Neurological Surgery, Neurology, and Pathology (Neuropathology), and the

Neurotransplantation Research Program, University of Pittsburgh, Pennsylvania

\begin{abstract}
Cellular therapy has been evaluated in small animals, subhuman primates, and now humans for the potential repair of brain injury due to stroke. Experimental striate stroke models have proven useful for the purpose of evaluating different treatment paradigms. Early clinical trials involving neuronal transplantation in patients suffering motor-related stroke in the basal ganglia region have begun.

This research will be described in this report.
\end{abstract}

KEY WORDS • stroke • transplantation • clinical trial • infarction

Modern restorative neurosurgery began more than 25 years ago when neurosurgeons and biologists envisioned the possibility of replacing human neurons in neurodegenerative diseases such as PD and HD. Early clinical trials were based first on a direct approach targeting the replacement of missing specific neurotransmitters rather than regenerating the damaged neuronal circuitry. More recently, because of the advent of therapeutic strategies derived from experimental work with stem and progenitor cells, there is hope that the final goal of reconstructing neuronal pathways may be achieved. We believe that cerebral infarction is an appropriate disorder to study. Dead neurons must be replaced, the grafts able to release neurotransmitters, and circuits have to be rebuilt. Of course, all these goals can be fulfilled only if our understanding of molecular mechanisms of disease keep pace with the development of new bioengineering strategies.

The field of neural transplantation for the treatment of neurological diseases became a potential therapy in 1979 when Bjorklund and Stenevi ${ }^{4}$ and Perlow, et al., ${ }^{29}$ showed that transplantation of dopamine-containing neurons in rat striatum improved functional deficits induced by damage to the nigrostriatal pathway. Since that time, neural transplantation has evolved from a therapy performed in the

Abbreviations used in this paper: $\mathrm{BDNF}=$ brain-derived neurotrophic factor; CNS = central nervous system; GDNF = glialderived neurotrophic factor; $\mathrm{HD}=$ Huntington disease NTF $=$ neurotrophic factor; $\mathrm{PD}=$ Parkinson disease. animal model to one applied in the human model with varying degrees of success. Animal models encompass a wide variety of disease states from degenerative diseases to trauma and stroke models. Types of tissue used for transplantation range from fetal tissue to tumor lines to stem cells. In some models, implants provide a source of neurotrophic factors. Various successes in animal models have led to the initiation of transplant trials in the humans. Clinical trials include transplantation in PD, HD, spinal cord injury, and stroke. As research in animal models progresses, transplant trials may be initiated for the treatment of multiple sclerosis, traumatic brain injury, cerebral palsy, amyotrophic lateral sclerosis, Alzheimer disease, hereditary ataxias, and other disorders.

\section{BACKGROUND OF NEURAL TRANSPLANTATION}

The concept of using dissociated CNS tissues for transplantation was promoted by Schmidt, et al. ${ }^{35}$ who argued that this protocol could be used for intraparenchymal implantation. The same group of investigators later provided detailed methodology and reported that mesencephalic dopaminergic-rich cell suspensions can be injected in various regions of lesioned rat brains and promote regeneration. ${ }^{5,13}$ Since these early attempts, a whole field has grown largely based on the idea of using human fetal tissues to replace human degenerating neurons. Investigation of PD has become the frontier in neurotransplantation. The hypotheses tested and lessons learned from 
transplantation studies in PD have been extended to other conditions such as HD and spinal cord regeneration. Among the challenges associated with any transplantation protocol are source and preparation of donor tissue, surgical protocol, posttransplantation hypoxia, local toxic factors such as free radicals and excitatory amino acids, deprivation of NTFs, necrosis, and neuronal apoptosis. Any factors found to alleviate these limitations will have a significant impact on the cell transplantation protocol in any neurodegenerative disease. The use of frozen cellular preparations in our human stroke trials has enabled us to have a shelf-life of the graft tissue.

\section{FUNDAMENTAL ISSUES}

\section{Tissue Availability}

An important issue in brain cell transplantation is the availability and suitability of tissues obtained in aborted fetuses. Despite the obvious ethical concerns, basic scientific investigations and clinical studies are still in progress, and many questions have yet to be answered. For example, in an extensive study of five tissue banks funded by the National Institutes of Health, only seven of approximately 1500 embryonic donors were considered suitable for transplantation in patients with PD. ${ }^{8}$ The authors of more recent studies have shown that the use of multiple donors or tissues obtained in first-trimester aborted fetuses are not absolute requirements to generate long-term survival of human brain grafts. Furthermore, of great promise is the current work with stem cells, neuronal precursors, and cell lines that may soon replace the use of primary human brain cells as the main donors. The value of cryopreservation has been questioned by some who argue in favor of in vitro processing. Longer-term graft preservation has remained a problem. Those involved in the University of Pittsburgh stroke transplantation trial were the first to use a cryopreserved cell line in patients. Another approach is to maintain fetal cells as aggregates in longterm suspension cultures that may even allow their expansion. ${ }^{37}$ This approach, however, may not be feasible for processed neuronal cell lines.

There are benefits to preimplantation in vitro processing of brain cells. First, processing may select for a viable better-characterized neuronal donor population. Second, it can help identify the donor glial-neuronal interactions that are critical in vivo, potentially protecting the graft from detrimental host reactions. Third, it can offer a setting for trophic and genetic manipulations that can enhance in vivo survival and integration. Finally, neuronal apoptosis in fetal grafts is well described and appears to be significant during the first 10 to 15 days postimplantation. ${ }^{23}$ The mechanisms of neuronal death are predominantly mediated by caspases, and inhibitors of this process may have important benefits in improving the viability of the grafts. $^{34}$

\section{Immunosuppression and Graft Success}

Investigators in current clinical trials have explored the need for immunosuppression and the relative contribution of the graft compared with the host response in promoting cell grafting. In 1991 the theory that immunosuppression may not be necessary was tested in a landmark study by Henderson, et al. ${ }^{17}$ In 12 patients with advanced PD in whom grafts were implanted in the caudate nucleus, these authors found long-term clinical benefits in the absence of immunosuppression. Recent work has shown successful tissue grafting without immunosuppression. Most agree that xenografts require long-term immunosuppression. The role of immunosuppression in transplantation after stroke has not been evaluated fully. We have used limited (2-month) or longer (6-month) periods of immunosuppression after cellular implantation in cases of stroke.

Based on various clinical trials involving fetal mesencephalic tissues, one of the most interesting observations is that the grafts appear to survive for long periods of time even if the underlying disease affecting the host dopaminergic neurons does not change its course. When we overcome problems in generating reliable, high-quality, thoroughly assessed grafting material, cell transplantation could become a standard adjuvant to drug therapy or deep brain stimulation in patients with PD.

It has become increasingly clear that the reaction of the host parenchyma to the fetal graft is the decisive factor in determining its survival, integration, and functional benefit. Since the initial studies involving murine models of neurotransplantation, investigators have suspected that part of the functional benefit may be due to the host's reaction to the graft or to surgical manipulation. Not surprisingly, growth and trophic factors or other molecules produced by the host reactive neuroglial millieu are prime candidates as mediators of these effects. The effects of the host-guided migration of donor-derived neurons to selective targets have been convincingly demonstrated. ${ }^{9}$ Nonetheless, the opposite may be as important clinically; that is, host sprouting in a dopaminergic graft may be specific and extensive. The most intriguing observations were made in the 1-methyl-4phenyl-1,2,3,6-tetrahydropyridine model of PD. Bankiewicz and colleagues ${ }^{3}$ have demonstrated in a long-term study of hemiparkinsonian monkeys that cavitation alone leads to similar clinical improvement as implantation of an autograft composed of dopaminesecreting adrenal medullary chromaffin cells. Furthermore, they reported similar results following implantation of fetal amnion. Results of both studies suggest strongly that the regenerative capacity of the host brain plays a primary role in functional recovery, at least in this primate model of PD.

Among the most prominent but least understood reactions of the host to the graft is the microglial response. It is known that reactive microglia are important immune mediators of antigen-presenting cells that infiltrate the grafted tissues. ${ }^{31}$ Still, the authors of several recent reports have suggested that their presence and less-defined activities are critical for the survival of the graft and host brain regeneration. Microglia react to injury, usually by responding to and producing NTF. ${ }^{10}$ Furthermore, other growth factors produced by microglia, such as hepatocyte growth factor, have been shown to have neurotrophic activities in the CNS..$^{15}$ Finally, in a direct experiment, activated macrophages transplanted into an injury site within the CNS were crucial in triggering a regenerative cascade. In vivo, nigrostriatal dopaminergic neurons develop neurites around the wound or graft, and this process appears to correlate with production of NTF by activated 
macrophages and microglia. For these reasons, the supportive role of the surrounding host neuroglial environment and associated neurotrophic support are crucial to promoting graft survival and differentiation.

\section{CEREBRAL INFARCTION}

Transplantation of human neuronal cells is a new approach for ameliorating functional deficits caused by CNS disease or injury. Several investigators have evaluated the effects of transplanted fetal tissue, rat striatum, or cellular grafts into small-animal stroke models. ${ }^{7}$ One of the best studied models of brain ischemia is the murine hippocampal stroke that results in well-defined lesions, especially in the CA1 region. The standardization of this model is invaluable to the reliable testing of various experimental protective and regenerative therapies. Among them, investigations involving cell transplantation of fetal hippocampal neurons have determined that they can survive and integrate in the ischemic brain. ${ }^{2}$ Methodological issues remain to be resolved because the authors of subsequent studies have questioned the capacity of rat fetal neocortical tissues, implanted in an infarcted area, to integrate in the surrounding host tissue..$^{14}$ It has been shown, however, that the chronic ischemic region can support graft tissue.

Because the widespread clinical use of primary human tissue is likely to be extremely limited as a result of ethical and logistical difficulties inherent in obtaining large quantities of fetal neurons, there has been considerable effort to develop alternate sources of human neurons for use in transplantation. One alternate source is the Ntera 2/cl.D1 human embryonic carcinoma-derived cell line. These cells proliferate in culture and differentiate into pure postmitotic human neuronal cells (LBS-Neurons; Layton BioScience, Sunnyvale, CA) upon treatment with retinoic acid. ${ }^{30,39}$ Thus, Ntera 2/cl.D1 precursor cells appear to function as CNS progenitor cells, possessing the capacity to develop diverse mature neuronal phenotypes. When transplanted, these neuronal cells survive, extend processes, express neurotransmitters, form functional synapses, and integrate with the host..$^{1,19}$ The final product is greater than $95 \%$ pure populations of human neuronal cells that appear virtually indistinguishable from terminally differentiated postmitotic neurons..$^{40}$ The cells are capable of differentiation to express different neuronal markers characteristic of mature neurons, including all three neurofilament proteins (subunits $\mathrm{L}, \mathrm{M}$, and $\mathrm{H}$ ); microtubuleassociated protein 2, the somal/dendritic protein; and tau, the axonal protein. Their neuronal phenotype makes them a promising candidate for replacement in CNS disorders as a virtually unlimited supply of pure postmitotic, terminally differentiated human neuronal cells. In support of different mechanisms for efficacy, the authors of animal studies in which LBS-Neurons were transplanted have demonstrated graft survival, mature neuronal phenotype, and integration into host brain in vivo. ${ }^{40}$ Because these cells derived from a cancer cell line, safety testing in rodent and primate models was imperative before instituting clinical trials. No evidence for malignant transformation has been identified in any experiment involving this cell line.
In patients disabled by stroke, the concept of restoring function by transplanting human neuronal cells into the brain is novel. ${ }^{6}$ Researchers studying a rat model of transient focal cerebral ischemia have demonstrated that transplantation of fetal tissue restored both behavioral and motor functions. ${ }^{28}$ Borlongan and colleagues ${ }^{6,7}$ were the first to show that LBS-Neuron graft could also reverse the deficits caused by stroke. Preclinical studies of LBSNeurons were conducted in a model of transient focal, rather than global, ischemia to maximize the chances of functional recovery. In animals implanted with LBS-Neurons (and that received cyclosporine treatment), amelioration of ischemia-induced behavioral deficits was evident throughout the 6-month observation period. The authors reported demonstrated recovery in the passive avoidance test, as well as recovery of motor function in the elevated body swing test. In comparison, in animals in control groups receiving transplants of rat fetal cerebellar cells, medium alone, or cyclosporine, no significant behavioral improvement was demonstrated. In a second study in which they evaluated response in comparison with the number of cells transplanted, the authors confirmed the efficacy of transplanted LBS-Neurons in reversing the behavioral deficits resulting from transient ischemia in a middle cerebral artery occlusion rat model.

The initial objective of the first 12-patient clinical study performed at the University of Pittsburgh was to demonstrate the safety and feasibility of the neuronal cell implantation procedure. ${ }^{20,38}$ This goal was met; no implantrelated adverse events have occurred in at least 24 months of follow up. The adverse events that did occur in these patients were thought to be unrelated to the implantation of the neuronal cells and can be considered typical of a population with known cardiovascular disease and advanced age. This study was also intended to provide some information on the efficacy of neuronal cell implantation in improving stroke-related neurological deficits. In both treatment groups, mean National Institutes of Health Stroke Scale total scores decreased and mean European Stroke Scale total scores increased; both changes indicated improvement. With regard to European Stroke Scale scores, the increases tended to be larger in the group of four patients receiving six million cells, both in the total scores and in the composite motor subscale scores. Both the Barthel Index and the Short Form-36 scores decreased in the group receiving two million cells and increased in the group receiving six million cells. All outcome measurements were consistent in identifying a trend toward improved scores in the group of patients in whom six million neuronal cells were transplanted. The results of ${ }^{18} \mathrm{~F}$ fluorodeoxyglucose-positron emission tomography studies also provided a suggestion of efficacy, in that increased activity at the area of the stroke was seen in six patients, including improvement in contralateral cerebellar hypometabolism. ${ }^{20,26}$ On autopsy examination performed 27 months following cell implantation in one patient, persistence of grafted neuronal cells at the target was observed. ${ }^{27}$ This patient had died of an unrelated medical illness. The graft site did not show signs of inflammation. This patient had experienced no clinical improvement after transplantation.

Neuronal cell implantation could improve neurological function through a number of different mechanisms: 
provision of neurotrophic support (acting as local pumps to support cell function), provision of neurotransmitters, reestablishment of local interneuronal connections, cell differentiation and integration, and improvement of regional oxygen tension. The LBS-Neurons release high levels of glial-derived NTF after transplantation into rats with stroke (Borlongan, et al., personal communication). Transplanted cells also may act to limit the reactive glial response as well as retrograde degeneration, although this may be less feasible in cases of chronic injury. We believe that axonal reconnections established through the grafted cells (serving as a "bridge") over large distances is less likely, although this phenomenon has been observed in spinal cord injury models. A Phase II dose-response trial was completed recently in which the authors evaluated further the role of neurotransplantation in patients with chronic motor deficits caused by infarction in the basal ganglia region.

At present, we are designing a third clinical trial to evaluate factors such as patient age as well as the need for immunosuppression on cellular implantation for basal ganglia stroke. Cell therapy has not yet become a treatment available to patients outside of research protocols. Our current inclusion criteria remain for basal ganglia stroke, although work is progressing in an evaluation of the safety of cortical transplantation. It is important that investigators in future trials compare implant-treated patients with control patients in whom no implant is placed. The natural history of stroke is poorly documented. Because no real treatment has been available, few patients have been systematically followed over extended time periods. In all patients enrolled in our studies stability and no improvement in their clinical condition in the months prior to randomization had been documented.

\section{DELIVERING CELLULAR SUPPORT}

In our opinion, the most important factor deciding the fate of the future graft is the trophic support offered by the intrinsic milieu, the host environment, or administered in vivo postgrafting. Neurotrophic factors have been the focus of intense studies in which they have been discussed as therapeutic agents in neurodegenerative diseases. ${ }^{16}$ Growth factors are crucial in the differentiation of neural progenitor cells or, in general, less differentiated cells that are abundant in the fetal grafts. Hepatocyte growth factor is only one of the newer trophins for the CNS. Today, the family of growth and trophic factors proposed to affect the survival and development of neuroprogenitor cells is probably the largest in this ever-expanding field. Factors such as cytokines, once considered to be exclusively neurotoxic (for example, transforming growth factor) are now studied for neurotrophic properties. Among them, leukemia inhibitory factor and ciliary NTF, in addition to more traditional growth factors such as platelet-derived growth factor, are considered to be potent promoters of neuroprogenitor cell proliferation and eventually differentiation. ${ }^{24}$ In vivo, reactive astrocyte-induced ciliary NTF can prevent the degeneration of dopaminergic neurons in adult rats. Astrocytes and endothelial cells surrounding or infiltrating the transplant are susceptible to the effects of platelet-derived growth factor, which may control the survival of graft through neovascularization.
Brain-derived neurotrophic factor has been shown to have marked potency in modulating the growth and survival of dopaminergic cells and their precursors. It is now widely accepted that the pluripotent BDNF and its highaffinity receptor trkB are widely distributed both in the developing and mature nervous system. Among the most exciting new discoveries in the field of NTF research is the ability of BDNF to be transported in an anterograde fashion. It was shown, for example that in development, BDNF produced by dorsal interneurons stimulates the proliferation and differentiation of motor neuron progenitors after anterograde transport. ${ }^{18}$ One of the most important implications of anterograde transport of BDNF is probably its participation in synaptic transmission and its role in postsynaptic differentiation. Currently, BDNF is most useful in cell transplantation to promote dopaminergic differentiation preimplantation.

Glial-derived neurotrophic factor has similar or even enhanced trophic functions on dopaminergic neurons and their precursors. In vivo, GDNF has also been shown to be relatively potent in animal models of dopaminergic protection or regeneration; however, results in human clinical trials have not been so encouraging. ${ }^{21}$ Nonetheless, its potential use in PD has made GDNF the prime candidate for neurotrophic treatments in association with fetal mesencephalic transplants of dopaminergic neurons. A related member of the transforming growth factor- $\beta$ family, GDNF was first identified and characterized in 1993. The authors of in vivo studies involving fetal mesencephalic grafts showed that injections of GDNF in the vicinity of the implant-treated rat brain resulted in significantly increased survival and growth of tyrosine hydroxylase-positive cells accompanied by marked functional improvement. ${ }^{32}$ As previously noted, the neuronal cells used in our stroke studies have been shown to release GDNF, which may represent a primary effect of the cell transplants.

\section{Stem Cells and Neuronal Precursors}

Recent advances in our ability to manipulate embryonic stem cells have created a new spectrum of potential therapies in tissue regeneration in general and brain in particular. They have also reignited the interest in brain stem cells and neuroprecursors that show many analogies with the considerably better-characterized hematopoietic system. ${ }^{33}$ The definition of brain stem cells in relation to neural precursors is increasingly more precise and tends to delineate better the distinction between totipotent, multipotent, and lineage committed. Excellent reviews concerning the immense potential of stem cells in regeneration were recently published. ${ }^{11,41}$ Findings that stem cells are present not only in the developing brain but also in the adult brain have further contributed to the tremendous interest in the potential for CNS regeneration.

The authors of many early studies reported successful harvesting of progenitor cells from various regions of the brain (the subventricular zone being the most popular) that can be expanded in vitro and then differentiate in vivo into functional neurons. ${ }^{36}$ The challenge remains to identify better neural progenitor cells and understand the mechanisms of growth and differentiation from embryonic stem cells. An important characteristic of neuroepithelial stem cells, which were described more than a decade ago and 
are still widely used, is expression of the intermediate filament protein, nestin..$^{22}$ More recently, new markers of neuronal progenitor cells, conserved in their evolution and useful for early lineage selection, include Musashi-1 and an epitope recognized by the 2F7 monoclonal antibody. The mechanisms of progenitor cell maturation appear to be similar in various brain regions and seem to depend primarily on a cascade of signals mediated by specific combinations of growth factors ${ }^{25}$ and molecules (such as noggin) that can modulate their activity.

Regardless of their origin, fetal or adult, neural precursor cells show much promise in brain repair. ${ }^{12,37}$ They can survive and differentiate in the lesioned host brain, although it seems that their predominant differentiation in vivo, posttransplantation, is along the astrocytic lineage. Not surprisingly, the migration and differentiation of the grafted precursor cells is significantly influenced by local cues that may even overcome the in vitro manipulations. Nonetheless, the potential for in vivo survival of grafted neural precursor cells can be fully exploited when they are used as platforms for gene delivery or engineered to modulate the NTF environment in the host brain. Such work has not yet been performed in the setting of stroke, but we hope that appropriate clinical trials will be planned soon.

\section{CONCLUSIONS}

Tremendous achievements in neuroscience over the past three decades have provided a solid foundation for basic and clinical research in stroke neurotransplantation. Restorative neurosurgical procedures will likely include a combination of approaches to maximize patient outcomes. We believe that cerebral infarction and selected neurodegenerative disorders are appropriate initial candidates for this research.

\section{Disclosure}

Dr. Kondziolka serves as a consultant for Layton BioScience, Inc.

\section{References}

1. Andrews PW: Retinoic acid induces neuronal differentiation of a cloned human embryonal carcinoma cell line in vitro. Dev Biol 103:285-293, 1984

2. Aoki H, Onodera H, Yae T, et al: Neural grafting to ischemic CA1 lesions in the rat hippocampus: an autoradiographic study. Neuroscience 56:345-354, 1993

3. Bankiewicz KS, Palmatier M, Plunkett RJ, et al: Reversal of hemiparkinsonian syndrome in nonhuman primates by amnion implantation into caudate nucleus. J Neurosurg 81:869-876, 1994

4. Bjorklund A, Stenevi U: Reformation of the severed septohippocampal cholinergic pathway in the adult rat by transplanted septal neurons. Cell Tissue Res 185:289-302, 1977

5. Bjorklund A, Stenevi U, Schmidt RH, et al: Intracerebral grafting of neuronal cell suspensions. II. Survival and growth of nigral cell suspensions implanted in different brain sites. Acta Physiol Scand Suppl 522:9-18, 1983

6. Borlongan CV, Koutouzis TK, Jorden JR, et al: Neural transplantation as an experimental treatment modality for cerebral ischemia. Neurosci Biobehav Rev 21:79-90, 1997

7. Borlongan CV, Tajima Y, Trojanowski JQ, et al: Cerebral ischemia and CNS transplantation: differential effects of grafted fetal rat striatal cells and human neurons derived from a clonal cell line. Neuroreport 9:3703-3709, 1998

8. Branch DW, Ducat L, Fantel A, et al: Suitability of fetal tissues from spontaneous abortions and from ectopic pregnancies for transplantation. Human Fetal Tissue Working Group. JAMA 273:66-68, 1995

9. Brustle O, Maskos U, McKay RD: Host-guided migration allows targeted introduction of neurons into the embryonic brain. Neuron 15:1275-1285, 1995

10. Elkabes S, DiCicco-Bloom EM, Black IB: Brain microglia/macrophages express neurotrophins that selectively regulate microglial proliferation and function. J Neurosci 16: 2508-2521, 1996

11. Fuchs E, Segre JA: Stem cells: a new lease on life. Cell 100: $143-155,2000$

12. Gage FH: Mammalian neural stem cells. Science 287: 1433-1438, 2000

13. Gage FH, Bjorklund A, Stenevi U, et al: Intracerebral grafting of neuronal cell suspensions. VIII. Survival and growth of implants of nigral and septal cell suspensions in intact brains of aged rats. Acta Physiol Scand Suppl 522:67-75, 1983

14. Grabowski M, Johansson BB, Brundin P: Neocortical grafts placed in the infarcted brain of adult rats: few or no efferent fibers grow from transplant to host. Exp Neurol 134:273-276, 1995

15. Hamanoue M, Takemoto N, Matsumoto K, et al: Neurotrophic effect of hepatocyte growth factor on central nervous system neurons in vitro. J Neurosci Res 43:554-564, 1996

16. Hefti F: Neurotrophic factor therapy for nervous system degenerative diseases. J Neurobiol 25:1418-1435, 1994

17. Henderson BT, Clough CG, Hughes RC, et al: Implantation of human fetal ventral mesencephalon to the right caudate nucleus in advanced Parkinson's disease. Arch Neurol 48:822-827, 1991

18. Kawaja MD, Rosenberg MB, Yoshida K, et al: Somatic gene transfer of nerve growth factor promotes the survival of axotomized septal neurons and the regeneration of their axons in adult rats. J Neurosci 12:2849-2864, 1992

19. Kleppner SR, Robinson KA, Trojanowski JQ, et al: Transplanted human neurons derived from a teratocarcinoma cell line (NTera-2) mature, integrate, and survive for over 1 year in the nude mouse brain. J Comp Neurol 357:618-632, 1995

20. Kondziolka D, Wechsler L, Goldstein S, et al: Transplantation of cultured human neuronal cells for patients with stroke. Neurology 55:565-569, 2000

21. Kordower JH, Palfi S, Chen EY, et al: Clinicopathological findings following intraventricular glial-derived neurotrophic factor treatment in a patient with Parkinson's disease. Ann Neurol 46:419-424, 1999

22. Lendahl U, Zimmerman LB, McKay RD: CNS stem cells express a new class of intermediate filament protein. Cell 60: 585-595, 1990

23. Mahalik TJ, Hahn WE, Clayton GH, et al: Programmed cell death in developing grafts of fetal substantia nigra. Exp Neurol 129:27-36, 1994

24. Maina F, Klein R: Hepatocyte growth factor, a versatile signal for developing neurons. Nat Neurosci 2:213-217, 1999

25. McKay R: Stem cells in the central nervous system. Science 276:66-71, 1997

26. Meltzer CC, Kondziolka D, Villemagne VL, et al: Serial $\left[{ }^{18} \mathrm{~F}\right]$ fluorodeoxyglucose positron emission tomography after human neuronal implantation for stroke. Neurosurgery 49:586-592, 2001

27. Nelson PT, Kondziolka D, Wechsler L, et al: Clonal human (hNT) neuron grafts for stroke therapy: neuropathology in a patient 27 months after implantation. Am J Pathol 160: 1201-1206, 2002

28. Nishino H, Koide K, Aihara N, et al: Striatal grafts in the 
ischemic striatum improve pallidal GABA release and passive avoidance. Brain Res Bull 32:517-520, 1993

29. Perlow MJ, Kumakura K, Guidotti A: Prolonged survival of bovine adrenal chromaffin cells in rat cerebral ventricles. Proc Natl Acad Sci U S A 77:5278-5281, 1980

30. Pleasure SJ, Lee VM: Ntera 2 cells: a human cell line which displays characteristics expected of a human committed neuronal progenitor cell. J Neurosci Res 35:585-602, 1993

31. Poltorak M, Freed WJ: Immunological reactions induced by intracerebral transplantation: evidence that host microglia but not astroglia are the antigen-presenting cells. Exp Neurol 103: 222-233, 1989

32. Rosenblad C, Martinez-Serrano A, Bjorklund A: Glial cell linederived neurotrophic factor increases survival, growth and function of intrastriatal fetal nigral dopaminergic grafts. Neuroscience 75:979-985, 1996

33. Scheffler B, Horn M, Blumcke I, et al: Marrow-mindedness: a perspective on neuropoiesis. Trends Neurosci 22:348-357, 1999

34. Schierle GS, Hansson O, Leist M, et al: Caspase inhibition reduces apoptosis and increases survival of nigral transplants. Nat Med 5:97-100, 1999

35. Schmidt RH, Bjorklund A, Stenevi U: Intracerebral grafting of dissociated CNS tissue suspensions: a new approach for neuronal transplantation to deep brain sites. Brain Res 218: 347-356, 1981

36. Studer L, Tabar V, McKay RD: Transplantation of expanded mesencephalic precursors leads to recovery in parkinsonian rats. Nat Neurosci 1:290-295, 1998

37. Svendsen CN, Smith AG: New prospects for human stem-cell therapy in the nervous system. Trends Neurosci 22:357-364, 1999

38. Thompson T, Lunsford LD, Kondziolka D: Restorative neurosurgery: opportunities for restoration of function in acquired, degenerative, and idiopathic neurological diseases. Neurosurgery 45:741-752, 1999

39. Trojanowski JQ, Mantione JR, Lee JH, et al: Neurons derived from a teratocarcinoma cell line establish molecular and structural polarity following transplantation into the rodent brain. Exp Neurol 122:283-294, 1993

40. Trojanowski JQ, Kleppner SR, Hartley RS, et al: Transfectable and transplantable postmitotic human neurons: a potential "platform" for gene therapy of nervous system diseases. Exp Neurol 144:92-97, 1997

41. Weissman IL: Stem cells: units of development, units of regeneration, and units in evolution. Cell 100:157-168, 2000

Manuscript received September 17, 2002.

Accepted in final form October 18, 2002.

Address reprint requests to: Douglas Kondziolka, M.D., Department of Neurological Surgery, Suite B-400, UPMC, 200 Lothrop Street, Pittsburgh, Pennsylvania 15213. email: kondziol@ neuronet.pitt.edu. 